\title{
The Differences in Students' Cognitive Processes in Constructing Mathematical Conjecture
}

\author{
I Wayan Puja Astawa* \\ Jurusan Matematika, Universitas Pendidikan Ganesha, Indonesia \\ e-mail: puja.astawa@undiksha.ac.id
}

\begin{abstract}
Constructing mathematical conjectures involves individuals' unique and complex cognitive processes in which have not yet fully understood. The cognitive processes refer to any of the mental functions assumed to be involved in the acquisition, storage, interpretation, manipulation, transformation, and the use of knowledge. Understanding of these cognitive processes may assist individuals in constructing mathematical conjectures. This study aimed to describe the differences in students' cognitive processes in constructing mathematical conjecture which is based on their mathematical ability and gender through a qualitative exploratory research study. The research subjects consisted of six mathematics students of Universitas Pendidikan Ganesha, the representative of high, medium, and low mathematical ability and either genders, male and female, respectively. The data of cognitive processes were collected by task-based interviews and were analyzed qualitatively. The differences in students' cognitive processes in constructing mathematical conjectures were grouped into five distinct stages, namely understanding the problem, exploring the problem, formulating the conjecture, justifying the conjecture, and proving the conjecture. The results show that there were several differences in the students' cognitive processes in constructing mathematical conjectures in the previously mentioned stages.
\end{abstract}

Keywords: Cognitive Process; Mathematical Conjecture; Level of Mathematical Ability; Gender

\section{Introduction}

A mathematical conjecture is a mathematical statement in which the truth needs to be proved (Ponte, 1998; Pedemonte, 2001; and Norton, 2000). This means that the truth of the mathematical conjecture cannot be determined directly without a clear evidence. Mathematical conjectures possess a pivotal role in mathematics for allowing the science to grow and develop (Mazur, 1997). Apart from mathematics, constructing mathematics conjecture also plays an important role in learning mathematics. Constructing mathematical conjectures holds an important position in mathematics instruction due to the three benefits it offers: it demonstrates learners' conception, plays an important role in reasoning, and helps with mathematics learning progression (Lim et al., 2010).

Constructing conjectures in the mathematics learning and instruction is an important research topic. Most of the research on conjecture studies on how individuals construct conjectures and examines the conjectures constructed by others (Jiang, 2002). Constructing mathematical conjectures involves complex cognitive processes (Ponte et al., 1998, Morseli, 2006; Canadas et al., 2007; and Astawa et al., 2018). Experts have provided varying descriptions on individuals' cognitive processes in constructing mathematical conjectures. Ponte et al. (1998) mentioned three stages in the cognitive processes involved in constructing a mathematical conjecture, namely proposing questions and formulating a conjecture, testing and refining the conjecture, and arguing and proving the conjecture. Meanwhile, Morseli (2006) mentioned four stages of cognition involved in constructing a conjecture, namely exploring the problem to find out a property, formulating and communicating a conjecture, exploring the conjecture and discovering the theoretical arguments that validate it, and construcing a proof for the conjecture that must be acceptable to the community of mathematicians. Canadas et al. (2007) elaborated on the stages of

\footnotetext{
"Corresponding author.

Received 6 September 2019; Accepted 13 March 2020; Available online 31 March 2020 (c) 2020 JPI. All Rights Reserved
} 
cognition involved in conjecture construction based on induction. They specified the stages of cognition based on empirical induction from a finite number of discrete and dynamic cases. There are seven stages of cognition in constructing conjectures identified from the finite number of discrete cases: observing cases; organizing cases; searching for patterns; formulating a conjecture; validating the conjecture; generalizing the conjecture; and justifying the conjecture. On the other side, six stages of cognition are involved in constructing conjectures from a number of dynamic cases, namely manipulating a situation dynamically through continuity of cases, observing an invariant property in the situation, formulating a conjecture that the property holds in other cases, validating the conjecture, generalizing the conjecture, and justifying the conjecture. Meanwhile, Astawa et al. (2018) formulated the cognitive process involved in constructing conjecture into five stages, namely understanding the problem, exploring the problem, formulating the conjecture, justifying the conjecture, and proving the conjecture. The cognitive process in the stage of understanding the problem includes reading the problem and determining what is given and what is asked. The cognitive process in the stage of exploring the problem includes transforming the problem into drawings or graphs, manipulating the problem by using various drawings and graphs, finding an invariant property and pattern to be constructed as a conjecture by observing the changes arising in the drawings and graphs, specifying the property found to be constructed as a conjecture, and linking to relevant mathematical knowledge in identifying the property or pattern observed from the changes in the drawings or graphs. The cognitive process in the stage formulating a conjecture includes remembering the conjecture obtained from exploring the problem from which the conjecture is formulated, writing down the conjecture by referring to the results of the exploration of the problem, language, or form, and believing that the conjecture formulated is comprehensible to others. The cognitive process in the stage justifying the conjecture includes explaining the arguments for the conjecture, generalizing the conjecture, and recognizing the flaws and errors in the conjecture formulated or in the underlying arguments. And lastly, the cognitive process in the stage proving the conjecture includes realizing that the truth of the conjecture needs proving and disclosing the steps involved in proving the truth of the conjecture, selecting the type of proof of the conjecture according to the conjecture constructed, and establishing the proof of the conjecture.

Cognition, basic mathematical ability, and gender have long grabbed the attention of both psychologists and neurologists. Various studies in the fields of psychology and neurology have generally demonstrated differences between males and females in three primary abilities, namely verbal, spatial, and arithmetical abilities (Ardila et al., 2011). Generally, females are better in the verbal abilities (Blazhenkova and Kozhevnikov, 2009; Herlitz and Rehnman, 2008), while their male counterparts are superior in both spatial and arithmetical abilities (Geist and King, 2008; Unal et al., 2009; Yang and Chen, 2010; Weinstein \& Laverghetta, 2010). Benbow et al. (2000) added another finding that males and females also differ in mathematical reasoning ability. However, males' superiority over females in particular cognitive tasks is inconsistent and constantly becomes subject to be debated. In terms of task completion ability, Scafidi and Bui (2010) stated that males and females are equal. Potur and Barkul (2009) added that the two groups also share a similarity in creative thinking, while Turgut and Yilmaz (2012) argued that males' spatial ability is not different from females' spatial ability.

Belnap and Parrot (2013) investigated mathematical conjecture with gender and mathematical ability taken into account. They looked at the conjectures constructed by the research subjects based on five dimensions. One of the dimensions is the cognitive processes the subjects went through when completing the task. However, they did not explicitly shed light on the differences in the cognitive processes involved in constructing conjectures based on the gender and mathematical ability of the subjects. In this article, the differences are clearly discussed

\section{Method}

This research was a qualitative exploratory study involving six subjects. The six subjects were students of Mathematics Education Study Program, Universitas Pendidikan 
Ganesha, who were vary in levels of mathematical ability and gender. The level of mathematical ability of students was determined by a mathematical ability test in the form of essay test which consisted of 10 items equivalent to the national selection test for new students. The subjects were coded $\mathrm{MH}$ (male of high level of mathematical ability), MM (male of medium level of mathematical ability), ML (male of low level of mathematical ability), FH (female of high level of mathematical ability), FM (female of medium level of mathematical ability), and FL (female of low level of mathematical ability). The data were collected by taskbased interviews. The tasks used in the data collection was the tasks of constructing conjectures from a geometric related to a quadrilateral which was formed by the angle bisectors of a parallelogram (see, Astawa, et.al. 2018).

Collecting data on students' cognitive processes in constructing mathematical conjecture consisted of two stages. In the first stage, all subjects were given the task to constructing mathematical conjecture on the assignment sheet according to the assigned task. Having constructed mathematical conjecture from the task, students were interviewed based on their works to obtain their cognitive processes in constructing the resulting mathematical conjecture. Both of these steps were carried out twice at different times. In the second repetition, the tasks formulation was modified by altering the symbols used to make them comparable.

The data obtained from the interviews were analyzed by Miles \& Huberman's qualitative data analysis technique (1994), which is comprised of data reduction, data presentation, and conclusion drawing. Triangulation was carried out based on the results of the first and second interviews to obtain credible data. The cognitive processes of every research subject were classified based on the cognitive processes proposed by Astawa et al. (2018), and the differences in the cognitive processes between subjects were then specified.

\section{Result and Discussion}

The cognitive processes of $\mathrm{MH}, \mathrm{MM}, \mathrm{ML}, \mathrm{FH}, \mathrm{FM}$ and $\mathrm{FL}$ in constructing mathematical conjectures based on the analysis of the interview and triangulation results are as follows. $\mathrm{MH}$ and $\mathrm{MM}$ showed all cognitive processes in constructing mathematical conjecture proposed by Astawa, et.al (2018). Meanwhile, ML did not show two processes in constructing mathematical conjecture which were the process of manipulating the problem by using drawings or graphs that reflect certain cases of the problem and the process of recognizing the flaws or errors in the conjecture formulated or the underlying arguments. $\mathrm{FH}$ did not show the process of recognizing the flaws or errors in the conjecture formulated or the underlying arguments either. FM and FL did not show all the cognitive processes in constructing mathematical conjecture either. Neither FM nor FL did not show the process of manipulating the problem by using drawings or graphs that reflect certain cases of the problem and the process of generalizing the conjecture. The cognitive processes of $\mathrm{MH}, \mathrm{MM}, \mathrm{ML}, \mathrm{FH}, \mathrm{FM}$ and $\mathrm{FL}$ in constructing mathematical conjectures is tabled completely in Table 1.

Table 1. Students' Cognitive Processes in Constructing Mathematical Conjectures

\begin{tabular}{|c|c|c|c|c|c|c|}
\hline \multirow{2}{*}{ Cognitive Processes } & \multicolumn{6}{|c|}{ Research Subjects } \\
\hline & MH & MM & ML & $\mathbf{F H}$ & FM & FL \\
\hline Reading the problem & $\sqrt{ }$ & $\sqrt{ }$ & $\sqrt{ }$ & $\sqrt{ }$ & $\sqrt{ }$ & $\sqrt{ }$ \\
\hline Determining what is given & $\sqrt{ }$ & $\sqrt{ }$ & $\sqrt{ }$ & $\sqrt{ }$ & $\sqrt{ }$ & $\sqrt{ }$ \\
\hline Determining what is asked & $\sqrt{ }$ & $\sqrt{ }$ & $\sqrt{ }$ & $\sqrt{ }$ & $\sqrt{ }$ & $\sqrt{ }$ \\
\hline Transforming the problem into drawings or graphs & $\sqrt{ }$ & $\sqrt{ }$ & $\sqrt{ }$ & $\sqrt{ }$ & $\sqrt{ }$ & $\sqrt{ }$ \\
\hline $\begin{array}{l}\text { Manipulating the problem by using drawings or graphs that reflect } \\
\text { certain cases of the problem }\end{array}$ & $\sqrt{ }$ & $\sqrt{ }$ & - & $\sqrt{ }$ & - & - \\
\hline $\begin{array}{l}\text { Finding an invariant property or pattern to be constructed as a } \\
\text { conjecture by observing the changes arising in the drawings or } \\
\text { graphs }\end{array}$ & $\sqrt{ }$ & $\sqrt{ }$ & $\sqrt{ }$ & $\sqrt{ }$ & $\sqrt{ }$ & $\sqrt{ }$ \\
\hline
\end{tabular}




\section{Cognitive Processes}

Research Subjects

Specifying the property obtained from the observation of the changes in the drawings or graphs to be constructed as a conjecture

Linking to relevant mathematical knowledge in identifying the property observed from the changes in the drawings and graphs Remembering the conjecture obtained from the exploration of the problem to be formulated into a conjecture

Writing down the conjecture by referring to the results of the exploration of the problem, language, and form

Believing that the conjecture is comprehensible to others

Explaining the arguments for the conjecture

Generalizing the conjecture

Recognizing the flaws or errors in the conjecture formulated or the underlying arguments

Realizing that the truth of the conjecture needs proving and disclosing the steps involved in proving the truth of the conjecture Selecting the type of proof of the conjecture in accordance with the conjecture constructed

Establishing the proof of the conjecture

$$
\sqrt{ }=\text { present }-=\text { absent }
$$

\subsection{The differences in the Cognitive Processes between Males and Females in Constructing Mathematical Conjectures}

The cognitive processes of male and female students of high level of mathematical ability ( $\mathrm{MH}$ and $\mathrm{FH}$ ) in constructing mathematical conjectures were relatively the same, but with some differing details, as shown in the snippets of the interviews with $\mathrm{MH}$ and $\mathrm{FH}$, particularly in the stage exploring the problem as shown in Exhibit 1 and Exhibit 2, respectively.

Exhibit 1. Snippet of the Interview with $\mathrm{MH}$

Code Questions from the Researcher $(R)$ and Answers from the Subject $(M H)$

$\mathrm{R} \quad$ : After finding out about the information, what did you do?

$\mathrm{MH} \quad$ : Firstly, I drew a number of parallelograms.
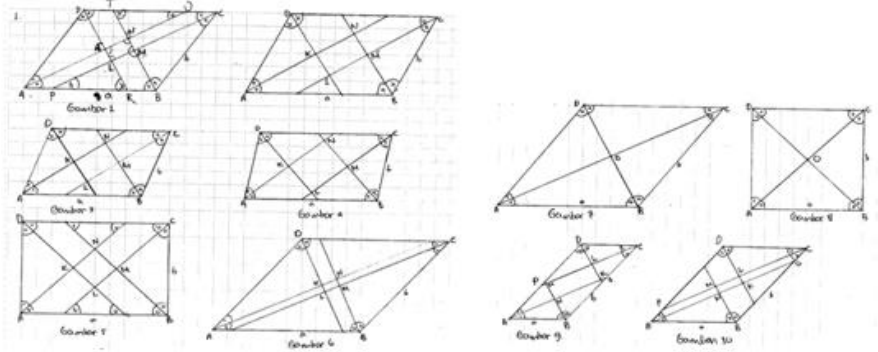

There were ten of them. I held the value of a constant and altered the value of $b$, and I found the quadrilateral KLMN to change in area.

$\mathrm{R} \quad$ : How did you set the values of $a$ and $b$ ?

$\mathrm{MH} \quad$ : The values of $a$ and $b$ were set randomly to get the parallelograms that I desired.

R : Why did you make many drawings?

$\mathrm{MH}$ : To see the form, the position, and what would happen to the quadrilateral KLMN area when the length of the sides were altered.

$\mathrm{R} \quad$ : What did you mean by seeing the form, position, and area?

$\mathrm{MH} \quad$ : On these drawings my conjectures would be based, for example, those relating to the form, position, and quadrilateral KLMN area. 
Exhibit 2. Snippet of the Interview with FH

Code Questions from the Researcher $(R)$ and Answers from the Subject (FH)

$\mathrm{R} \quad$ : What did you do then after gaining understanding of the problem?

$\mathrm{FH} \quad$ : After reading, I made a number of drawings.

$\mathrm{R} \quad$ : Would you like to show me the drawings?

$\mathrm{FH} \quad$ : Here, I drew some when $a$ was smaller than $b$ and some when $b$ was smaller than $a$.

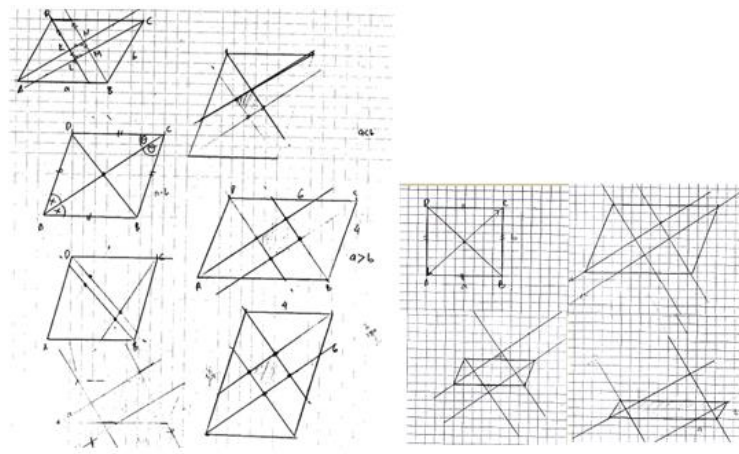

$\mathrm{R} \quad$ : Why did you make different drawings?

$\mathrm{FH} \quad$ : Because to make conjectures, it definitely takes creativity for me to be able to conceive the quadrilateral KLMN, like the form, position, and area.

$\mathrm{R} \quad$ : What did you do after making the drawings?

$\mathrm{FH} \quad$ : I observed the changes in the drawings in relation to the form, position, and area of the quadrilateral KLMN.

Some other differences in the cognitive processes between $\mathrm{MH}$ and $\mathrm{FH}$ in constructing mathematical conjectures are presented in Table 2.

Table 2. Differences in the Cognitive Processes between $\mathrm{MH}$ and $\mathrm{FH}$ in Constructing Mathematical Conjectures
Male of high level of mathematical ability (MH)
Female of high level of mathematical ability (FH)

\section{Exploring the problem}

Specifying the properties of the quadrilateral KLMN to be constructed as conjectures of the properties of the quadrilateral formed from the angle bisectors of a parallelogram in relation Specifying the properties of the quadrilateral KLMN that were constructed as conjectures of the quadrilateral formed from the angle bisectors of a to the form, position, and area. parallelogram in relation to the form and position.

\section{Formulating conjectures}

Formulating three conjectures, namely those related to the form, position, and area of the quadrilateral formed from the angle bisectors of a parallelogram.

Writing down conjectures in the form of Writing down conjectures in the form of implication, statements with universal quantifier and "If ..., then ..." and "... if ...." implication, "If ..., then ...."

\section{Justifying the conjectures}

Explaining the arguments for the conjectures by connecting them to the knowledge on the properties of the diagonal lines of a parallelogram and the properties of similarity and congruence in triangles.
Formulating two conjectures, namely those related to the form and position of the quadrilateral formed from the angle bisectors of a parallelogram.

Explaining the arguments for the conjectures by connecting them to the knowledge on the properties of rectangles, the properties of the diagonal lines of a rhombus, the properties of congruence in triangles, and the properties of the diagonal lines of a square. 
Male of high level of mathematical ability (MH)
Female of high level of mathematical ability

(FH)

\section{Proving the conjectures}

Proving the conjectures by drawing on the knowledge on the properties of similarity in triangles and the number of angular points in a quadrilateral as well as drawings.

Proving the conjectures by drawing on the knowledge on the properties of rectangles and the properties of the diagonal lines of a rhombus as well as drawings.

The cognitive processes of male and female of medium level of mathematical ability (MM and FM) are different. The differences were most apparent in the stage exploring the problem. The following are the snippets of the interviews with MM and FM in the stage exploring the problem.

Exhibit 3. Snippet of the Interview with MM

Code Questions from the Researcher $(R)$ and Answers from the Subject (MM)

$\mathrm{R} \quad$ : What did you do after understanding the problem?

MM : I started drawing.

$\mathrm{R} \quad$ : Drawing. Would you mind showing me the drawings?

MM : Here they are.
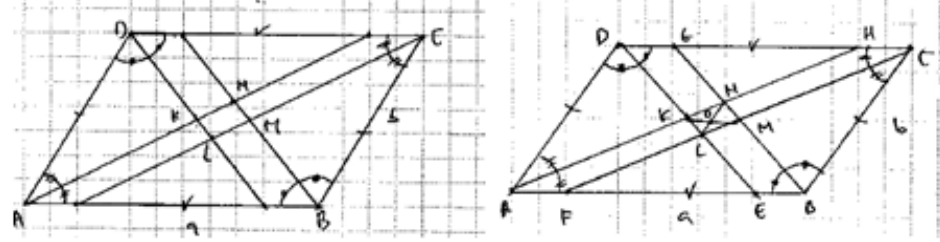

$\mathrm{R} \quad$ : You made some drawings. How many were there exactly?

MM : Only two of them.

$\mathrm{R} \quad$ : Did the drawings reflect all of the information regarding the problem?

MM : Yes.

$\mathrm{R} \quad$ : What was the difference in the two drawings you made?

MM : The drawings were consistent with the conjectures I made.

$\mathrm{R} \quad$ : So you made drawings first before making conjectures?

MM : Yes

Exhibit 4. Snippet of the Interview with FM

Code Questions from the Researcher $(R)$ and Answers from the Subject (FM)

$\mathrm{R} \quad$ : After gaining understanding on the problem, what did you do?

FM : After gaining the quadrilateral KLMN, I made some assumptions about it: the form, the position, the length of the sides, the area. For example, I made my first conjecture by making eight drawings, concluding that the measure of every angle of the quadrilateral KLMN was 90 degrees.
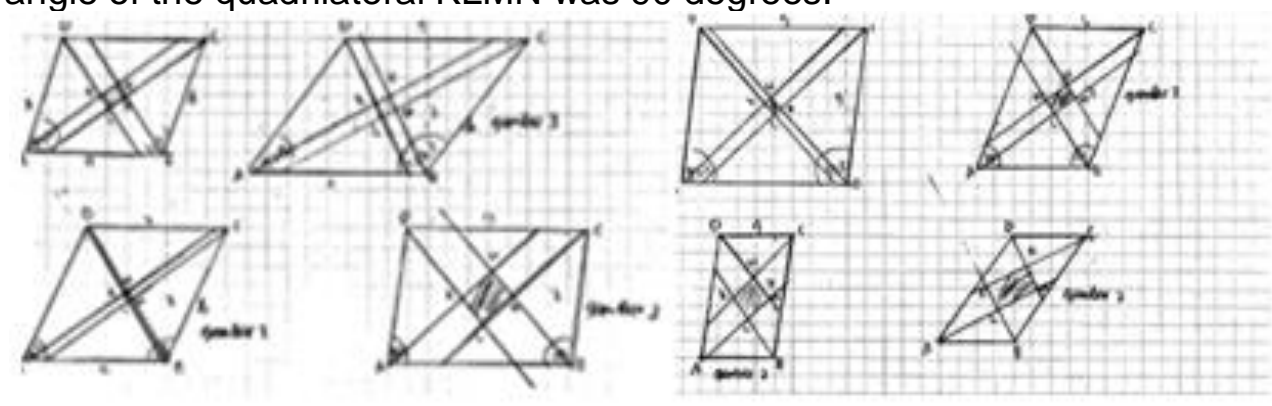


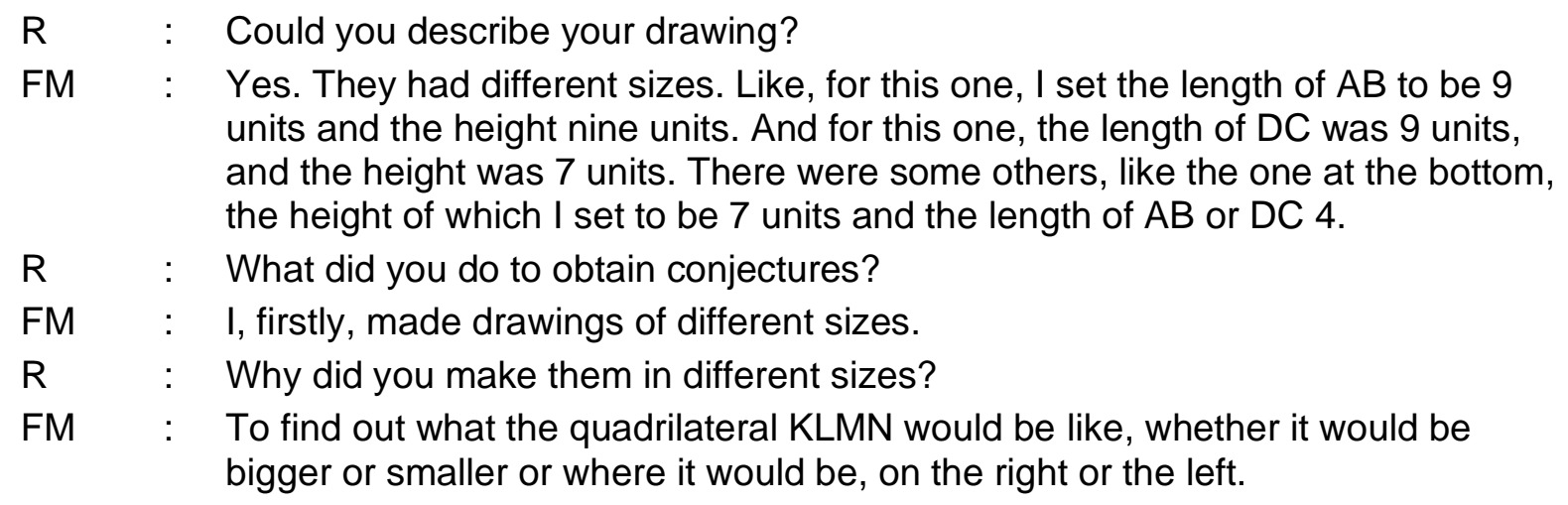

The other differences in the cognitive processes between MM and FM in constructing mathematical conjectures are presented in Table 3.

Table 3. Differences in the Cognitive Processes between MM and FM in Constructing Mathematical Conjectures

\begin{tabular}{cc}
\hline $\begin{array}{c}\text { Male of medium level of mathematical ability } \\
\text { (MM) }\end{array}$ & $\begin{array}{c}\text { Female of medium level of mathematical ability } \\
\text { (FM) }\end{array}$ \\
\hline
\end{tabular}

Selecting the types of conjectures to be constructed based on what was given in the problem.

Transforming the problem into drawings according to the conjectures constructed.

Transforming the problem into drawings of parallelograms in different sizes.

Not manipulating the problem with different Manipulating the problem by drawing cases, only making two drawings.

Finding invariant properties of the quadrilateral formed from the angle bisectors of a parallelogram to be constructed as conjectures by observing two drawings.

Specifying the properties of the quadrilateral formed from the angle bisectors of a parallelogram obtained by observing the drawings made to be constructed as conjectures. parallelograms of different sizes.

Finding invariant properties of the quadrilateral formed from the angle bisectors of a parallelogram to be constructed as conjectures.

Specifying the properties of the quadrilateral formed from the angle bisectors of a parallelogram obtained from investigating the changes in the drawings to be constructed as conjectures.

\section{Formulating Conjectures}

Formulating two conjectures, namely those on the form and position of the quadrilateral formed from the angle bisectors of a parallelogram.

Writing down conjectures implicitly in the form of statements with universal quantifier.

Believing that the conjectures were comprehensible to others as they were stated in simple language.

\section{Justifying the conjectures}

Conulating three conjectures, namely those on the angle/form, position, and length of sides/area of the quadrilateral formed from the angle bisectors of a parallelogram.

Writing down conjectures in the form of statements with universal quantifier and implication, "if ..., then ..."

Believing that the conjectures were comprehensible to others as they were accompanied with drawings.

Explaining the arguments for the conjectures by drawing on the knowledge on the properties of similarity in triangles, the number of angular points in a triangle, and concentric figures.

The process of generalizing the conjectures was undertaken without the manipulation of varying cases because there was only one parallelogram drawing which was made

Explaining the arguments for the conjectures by drawing on drawings, performing measurement, and connecting them to the mathematical knowledge on the properties of rectangles.

The process of generalizing the conjectures were undertaken by manipulation of varying cases by drawing parallelograms of different sizes. 


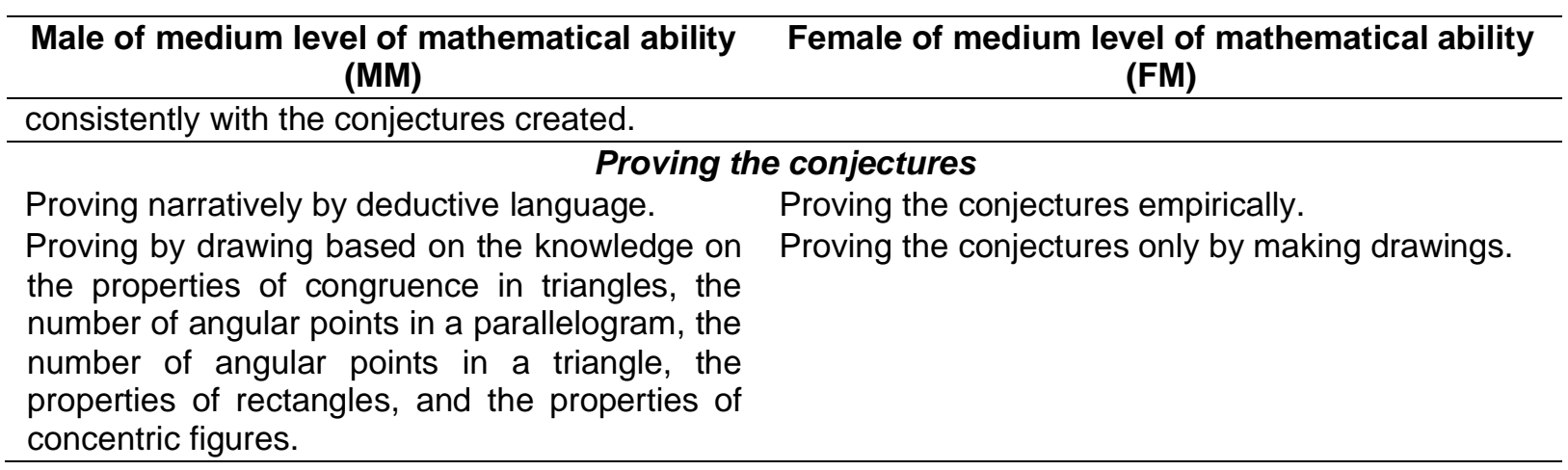

The cognitive processes of male and female of low level of mathematical ability (ML and $\mathrm{FL}$ ) were also different. The differences were most apparent in the stage exploring the problem. The following are the snippets of the interviews with $M L$ and FL in the stage exploring the problem.

Exhibit 5. Snippet of the Interview with ML

Code Questions from the Researcher $(R)$ and Answers from the Subject $(M L)$

$\mathrm{R} \quad$ : After understanding the problem, what did you do to obtain what was asked to find?

ML : Firstly, I made drawings.

R : Made drawings?

ML : Yes

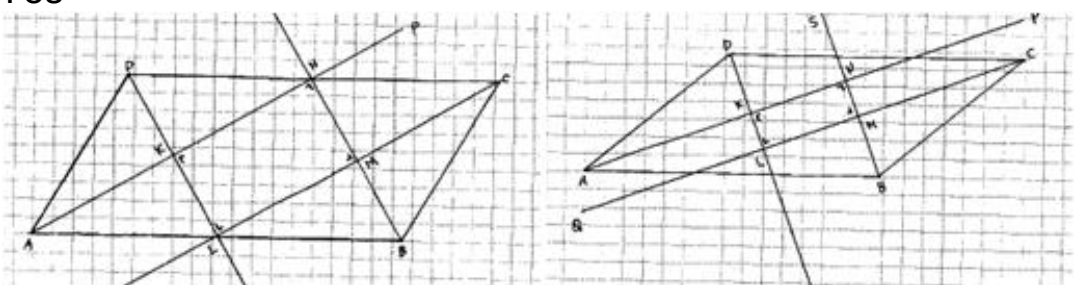

R : How many drawings did you make?

ML : Two.

R : With the two drawings you made, could you guarantee that your conjectures would apply to the parallelogram condition given in this problem?

ML : Yes

R : Why?

ML : Because any other drawings will also produce the same results.

Exhibit 6. Snippet of the Interview with FL.

Code Questions from the Researcher $(R)$ and Answers from the Subject ( $F L)$

$\mathrm{R} \quad$ : After finding out what was given and what was asked in the problem, what did you do?

FL : I drew.

$\mathrm{R}$ : Could you show me?

FL : Here.

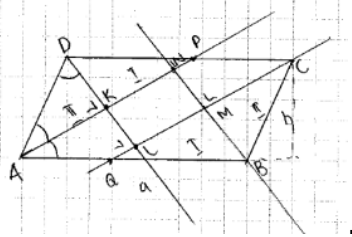

R : How many drawings were there?

FL : Only one. 
$\begin{array}{lll}\mathrm{R} & : \text { What did you do to make conjectures? } \\ \mathrm{FL} & : \text { Based on these drawings I made my conjectures. } \\ \mathrm{R} & : \text { How did you made conjectures from that drawing? } \\ \mathrm{FL} & : \text { I took a look at the drawing then made my conjectures. }\end{array}$

The other differences in the cognitive processes between $\mathrm{ML}$ and $\mathrm{FL}$ in constructing mathematical conjectures are presented in Table 4.

Table 4. Differences in the Cognitive Processes between ML and FL in Constructing Mathematical Conjectures

Male of low level of mathematical
ability (ML) $\begin{gathered}\text { Understanding the problem } \\ \text { (FL) }\end{gathered}$

The results presented above indicate that the cognitive processes of male and female students of high level of mathematical ability in constructing mathematical conjectures were generally the same, and as well as those male and female students of low level of mathematical ability. The differences that arose lied in the outcomes of the cognitive processes, namely the conjectures constructed. Such differences were due to differences in attention between both genders and the recognition of mathematical properties while exploring the problem and formulating the conjectures. This was associated with the disparities between the verbal, spatial, and arithmetic knowledge of the subjects. The 
differences in the outcomes of the cognitive processes were noted by Ardila et al. (2011), Geist and King (2008), Unal et al. (2009), and Yang and Chen (2010), who stated that males and females differ in their verbal, spatial, and arithmetic abilities, as well as with the case in this research. On the other hand, the cognitive processes of male and female students of medium level of mathematical ability in constructing mathematical conjectures were different. The difference lied in the mental activity after they gained the understanding of the problem. The male student of medium level of mathematical ability chose or established conjectures to be constructed after understanding the problem. The conjectures to be constructed were chosen and established from what was asked in the problem. After choosing or establishing the conjectures to be constructed, he transformed the problem into drawings and graphs and then constructed conjectures based on those drawings or graphs right away. The differences in the cognitive processes of the two subjects were also found in Morseli's study (2006), which pointed out the differences in the cognitive processes profile between individuals in constructing conjectures as per the steps of conjecture construction

\subsection{The differences in the Cognitive Processes of Students of High, Medium, and Low Levels of Mathematical Ability in Constructing Mathematical Conjectures}

The cognitive processes of male students of high, medium, and low levels of mathematical abilities in constructing mathematical conjectures are varied. The male students of high and low levels of mathematical ability constructed conjectures by manipulating the problem before choosing information that was relevant to the conjectures to be constructed, while the male student of medium level of mathematical ability chose information that was relevant to the conjectures to be constructed first. The difference was also captured in the cognitive processes in making a connection to relevant knowledge to explain and prove the conjectures.

The cognitive processes of female students of high and medium levels of mathematical ability in constructing mathematical conjectures were relatively the same. They only differed in terms of what were constructed as conjectures, which affected them in choosing which knowledge they would use to explain the arguments for their respective conjectures and prove them. Meanwhile, the female student of low level of mathematical ability went through different cognitive processes from those of high and medium levels of mathematical ability in constructing mathematical conjectures. The difference was apparent when she was manipulating the problem and generalizing her conjectures. Aside from the differences aforementioned, female students of high, medium, and low levels of mathematical ability had different cognitive processes in finding and specifying the properties to be constructed as conjectures when exploring the problem as well. This difference led to differences in the conjectures constructed and the knowledge used to explain their arguments for their conjectures and prove them.

The results above indicate that students' cognitive processes in constructing mathematical conjectures are different according to their level of mathematical ability. In subjects of high level of mathematical ability, the cognitive processes in constructing conjectures were complete and logical, while in those of medium and low levels of mathematical ability, the cognitive processes were incomplete and less logical.

\section{Conclusion and Suggestion}

Based on the results and discussion above, the following two conclusions were drawn:

- The cognitive processes in constructing mathematical conjectures are different between students of high, medium, and low levels of mathematical ability. Those of high level of mathematical ability had cognitive processes that were complete and logical, while those of medium and low levels of mathematical ability had cognitive processes that were incomplete and less logical.

- The cognitive processes of male and female students of medium level of mathematical ability in constructing mathematical conjecture are different from those of the rest, while students of high and low levels of mathematical ability had no difference in their cognitive 
processes in constructing mathematical conjectures.

Based on the discussion, it is suggested to the teacher to give attention to the mahtematical ability of student carefully during his/her teaching of mathematics process, an activity of constructing mathematical conjecture is going to be implemented.

\section{References}

Ardila, A., Rosselli, M., Matute, E., Inozemtseva, O. (2011). Gender differences in cognitive development. Developmental Psychology. 47 (4), Jul 2011, 984-990

Astawa, I W. P, Budayasa, I K., \& Juniati, D. (2018). The Process of Student Cognition in Constructing Mathematical Conjecture. Journal on Mathematics Education, 9 (1), 1526

Belnap, J. K and Parrot, A.L. (2013). Understanding Mathematical Conjecturing. In S. Brown, G. Karakok, K. H Roh, and M. Oehrtman (Eds). Proceeding of the $16^{\text {th }}$ Annual Conferenceon Research in Undergraduate Mathematics Education. Feb 21-23, 2013. Denver: Colorado

Benbow, C.P., Lubinski, D., Shea, D. L., and Eftekhari-Sanjani, H. (2000). Sex Differences in Mathematical Reasoning Ability at Age 13: Their Status 20 Years Later. Pshycological Science, 11 (6), 474-480

Blazhenkova, O and Kozhevnikov, M. (2009). The New Object-Spatial-Verbal Cognitive Style Model: Theory and Measurement. Appl. Cognit. Psychol. 23, 638-663

Canadas, M, C., Deulofeu, J., Figueiras, L., Reid, D. and Yevdokimov, O. (2007). The Conjecturing Process: Perspectives in Theory and Implication in Practice. Journal of Teaching and Learning, 5 (1), 55-72

Geist, E. A \& King, M. (2008). Different, Not Better: Gender Differences in Mathematics Learning and Achievement. Journal of Instructional Psychology, 35 (1), 43-52

Herlitz, A. and Rehnman, J. (2008). Sex Differences in Episodic Memory. Current Direction in Psychological Science, 17 (1), 52-56

Jiang, Z. (2002). Developing preservice teachers' mathematical reasoning and proof abilities in the Geometer's Sketchpad environment. In Mewborn, D. S., Sztajn, P., White, D. Y., Wiegel, H. G., Bryant, R. L., \& Nooney, K (eds). 2002. Proceedings of the $24^{\text {th }}$ Annual Meeting of the North American Chapter of the International Group for thePsychology of Mathematics Education, 717-729. Columbus, OH: ERIC Clearinghouse for Science, Mathematics, and Environmental Education.

Lim, H. K., Buendia, G., Kim, O., Cordero, F., and Kasmer, L. (2010). The role of prediction in the teaching and learning of mathematics. International Journal of Mathematical Education in Science and Technology, 41 (5), 595 - 608

Mazur, B. (1997). Conjecture. Synthese, 111, 197-210

Miles, M. B. and Huberman, A. M. (1994). Qualitative Data Analysis $2^{\text {nd }}$ an Exanded Source book. California: Sage Publication

Morseli, F. (2006). Use of Examples in Conjecturing and Proving: An Exploratory Study. In Novotna, J., Moraova, K., Kratka, M., and Stehlikova, N. (eds). Proceeding 30 $0^{\text {th }}$ Conference of the International Group for the Psychology of Mathematics Educations, Vol. 4. 185-192. Prague: PME

Norton, A. (2000). Student Conjectures in Geometri. Paper presented at the 24th conference of the international group for the Psychology of Mathematics Education. Hirosima, Japan

Pedemonte, B. (2001). Some Cognitive Aspects of the Relationship between Argumentation and Proof in Mathematics". In van den Heuvel-Panhuizen M. Preceeding of the 25th conference of the international group for the Psychology of Mathematics Education. Utrech, Netherland

Ponte, J. P., Ferreira, C., Brunheira, L., Oliveira, H., and Varandas, J. (1998). Investigating Mathematical Investigation. In P. Abrantes, J. Porfirio, and M. Baia (Eds.). Les interactions dans la classe de mathematiques: Proceedings of the CIEAEM 49. Pp. 3-14. Setubal: Ese de Setubal 
Potur, A. A \& Barkul, O. (2009). Gender and creative thinking in education: A theoretical and experimental overview. ITU A/Z, 6 (2), 44-57

Scafidi, T dan Bui, K. (2010). Gender similarities in math performance from middleschool through high school. Journal of Instructional Psychology, 37 (3), 252-255

Turgut, M \& Yilmaz, S. (2012). Relationship Among Preservice Primary Teachers' Gender, Academic Success and Spatial Ability. International Journal of Instruction, 5 (2), 5 - 20

Unal, H., Elizabeth Jakubowski and Darryl Corey. (2009). Differences in learning geometry among high and low spatial ability pre-service mathematics teachers. International Journal of Mathematical Education in Science and Technology, 40 (8), 997-1012

Weinstein, L. and Laverghetta, A. (2010). Sex Differences in College Students' Elementary Arithmatic Ability. College Student Journal, Sept 201044 (3), 700-702

Yang, J. C dan Chen, S. Y. (2010). Effects of gender differences and spatial abilities within a digital pentominoes game. Computers \& Education, 55 (3), 1220-1233 